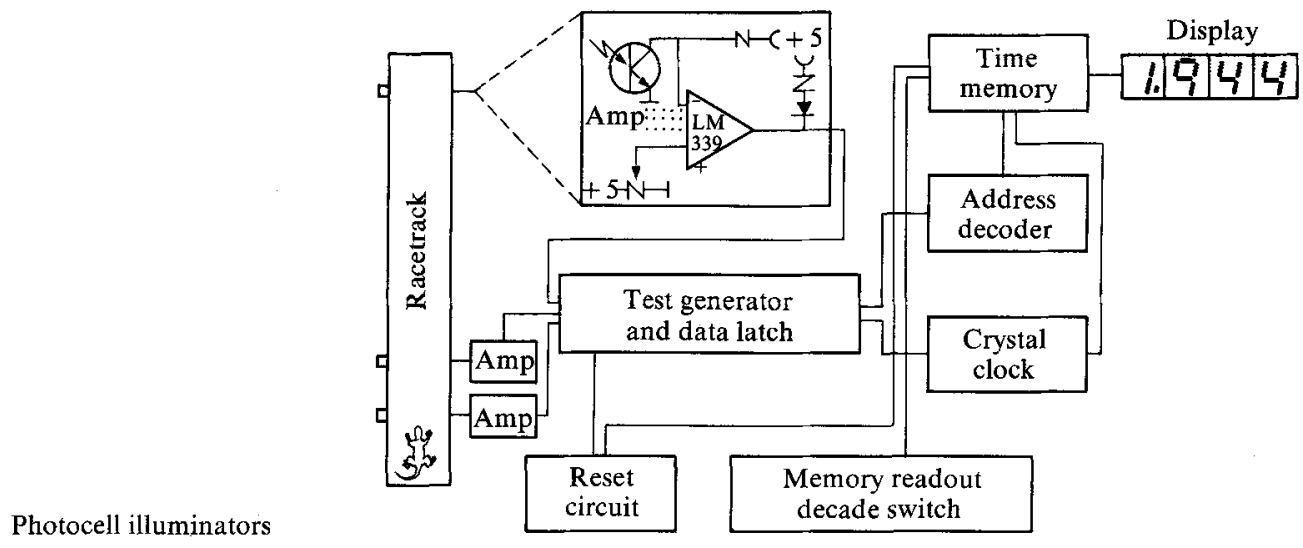

ry. The experimenter then manually recalls each time on a digital display and clears all registers for the next run. Elapsed times and distances can then be fitted to curvilinear equations to estimate acceleration and maximum velocity ${ }^{2}$.

The timer uses transistor-transistor logic (TTL), has a $1-\mathrm{MHz}$ crystal clock, and includes a self-test function (fig.). The timer is relatively stable (ours has drifted by only $30 \mathrm{ppm}$ in 14 months) and insensitive to ambient temperature ( $75 \mathrm{ppm}$ drift between 15 and $40^{\circ} \mathrm{C}$ ). Power can be either AC or DC (9-12 V). For our studies of lizards, the timer is adjusted to read from 0.001 to $9.999 \mathrm{sec}$. A maximum of 15 times can be stored in either cumulative or interval modes.

Each photocell station consists of a pre-focused bulb and 4 vertically aligned photo-transistors. Because an animal thus interrupts a 'plane' of light, the discrepancy for each time interval is minimized to the width of the light plane. (However, if only 1 photocell were used, the animal's nose might fail to break the beam, thereby potentially adding a substantial source of error to estimates of velocity or acceleration.)

To facilitate data storage and analysis, the photocell stations can be connected to an AIM-65 microprocessor (4K), which can be programmed to accept input from a maximum of 16 photocell stations. A control program (BASIC) activates a machine-language program (which records elapsed times) and then calculates and prints relevant data (e.g., species, animal identification number, elapsed times, interval times, and interval velocities). Data can also be stored on an audio-cassette or disk for detailed statistical analysis on a larger computer.

We have also connected the photocell stations to an Apple II + microcomputer (48K) using a Versatile Interface Adaptor (VIA, John Bell Engineering, Redwood City, California). Programs are modified as appropriate.

Results and discussion. The compact electronic package, battery power supply, stability, and thermal insensitivity permit the use of this timer in remote field situations for extended periods. Because times can be read immediately, delays and expense of processing and analyzing film are eliminated. Moreover, an experimenter can easily monitor the status of the apparatus as well as detect and capitalize on experimental results while still in a position to gather additional data.

1 This research was supported by NSF DEB 78-12124, the Miller Institute for Basic Research in Science (University of California, Berkeley), and by the Graduate School Research Fund (University of Washington, Seattle). The authors gladly acknowledge the advice of R. Reinstatler, J.R. Simpson, M.O. Smith, and especially A. M. Hawkins.

2 F.M. Henry and I. R. Trafton, Res. Q. Am. Ass. Hith phys. Educ. 22, 409 (1951).

\title{
Assay of aminoglycosides is influenced by tissue homogenization technique ${ }^{1}$
}

\author{
Carol S. Desrochers and J. Schacht
}

\section{Kresge Hearing Research Institute, University of Michigan, Ann Arbor (Michigan 48109, USA), 6 April 1981}

Summary. Recovery of neomycin from tissue homogenates was lower when glass/glass homogenizers were used due to the abrasion of glass which will bind to the drug. Glass homogenizers should be avoided for the determination of aminoglycoside levels in tissues.

Adhesion of aminoglycoside antibiotics to glass surfaces is a well known phenomenon. Instructions for assay of serum drug levels commonly include warnings that tests should not be carried out in glass tubes. Recent experiments in our laboratory indicate that aminoglycoside-glass interactions may pose a considerable problem in the analysis of tissue drug levels.

The analysis of body tissues requires prior homogenization. A random survey of 8 publications reporting drug levels in human and animal tissues showed that 7 indicated a homogenate was prepared by a non-specified procedure. 1 publication mentioned the use of glass-glass homogenizers. While studying neomycin levels in guinea-pig tissues we used 2 different homogenization techniques. For large body tissues, such as liver and kidney, a Polytron blender (Brinkman Instruments, Westbury, NY) was employed while small samples of inner ear tissues were prepared in a glass homogenizer. Homogenates were transferred to polypropylene tubes in which all subsequent manipulations were performed. 
Routine recovery experiments were done by adding known amounts of neomycin to tissue homogenates. After centrifugation for $2 \mathrm{~min}$ at $12,000 \times \mathrm{g}$ the supernatant fraction ${ }^{2}$ was assayed by a disc plate microbiological assay ${ }^{3,4}$. For liver, kidney and other body tissues recoveries were complete (table). Recovery from homogenates of inner ear tissues, however, was low and variable, $39 \pm 22 \%$ (range, $15-88 \%)$. To investigate the cause of the low yields buffer was 'homogenized' without added tissues in glass-glass homogenizers and transferred to polypropylene tubes. Recovery of neomycin from this buffer was similar as with the ear tissues, $42 \pm 21 \%$ (range, $21-55 \%$ ). As only the homogenization but not the subsequent assays were carried out in glass tubes it seems that preparation of tissue

Recovery of neomycin from homogenates

\begin{tabular}{llc}
\hline Tissue & Technique & $\begin{array}{l}\text { Neomycin } \\
\text { recovered (\%) }\end{array}$ \\
\hline Kidney & Polytron & $125 \pm 27$ \\
Liver & Polytron & $91 \pm 13$ \\
Inner ear & Glass/glass & $39 \pm 22$ \\
None, buffer only & Glass/glass & $42 \pm 21$ \\
\hline
\end{tabular}

Tissue homogenates were prepared in $0.2 \mathrm{M}$ sodium phosphate, $\mathrm{pH} 8$ and standard amounts of neomycin $(5-20 \mu \mathrm{g} / 100 \mu 1$ homogenate) were added. Inner ear tissues are a pool of stria vascularis, spiral ligament and organ of Corti. Numbers are means $\pm S D$. homogenates in glass homogenizers can lead to sufficient abrasion of glass to lower the yields of antibiotics. It is important to note that loss of drug during tissue preparation is detected only in recovery studies with the original homogenate because glass particles are removed by centrifugation. Drug standards generated with the supernatant fraction of ear tissue homogenates showed recoveries of 112 and $122 \%$.

A number of variables may influence recovery such as the amount of tissue in relation to the surface of the glass homogenizer; the concentration of the drug; the force with which homogenization is carried out; the use of glass or teflon pestles in connection with a glass tube; the affinity of the aminoglycosides for glass. From these considerations, it seems indicated to avoid glass homogenizers and to conduct recovery experiments by adding the aminoglycoside prior to the homogenization of tissues.

1 Acknowledgment. Supported by research grant NS-13792 and Program Project grant NS-05785 from the National Institutes of Health.

2 D.C. Grove and W. A. Randall, Assay methods of antibiotics: A laboratory manual. Medical Encyclopedia, Inc., New York 1955.

3 W.W. Davis, Appl. Microbiol. 22, 659 (1971)

4 W.W. Davis, Appl. Microbiol. 22, 666 (1971).

\section{CORRIGENDUM}

S. - C. Chen: A simple synthesis of (E)-3-formylbut-2-enenitrile, and its use as a precursor of isotope-labelled zeatin and $( \pm)$ dihydrozeatin, Experientia 37,543 (1981). On page
544 , line 19 from the bottom as well as in the figure (right hand side between formula 4 and 6 ) it should correctly read $-\mathrm{CoCl}_{2}$ instead of $-\mathrm{COCl}_{2}$.

\section{Instructions to authors}

Experientia is published on the 15th of every month and can be obtained in any country through booksellers or from the publishers. All communications to the editors should be addressed to the publishers. All manuscripts for publication in a given number must be in the hands of the editors 3 months before publication.

Articles of general scientific interest, of interdisciplinary character: briefly stated and hitherto unpublished original reports of sufficient novelty value. In no case will papers of preliminary nature be accepted.

Experiments Papers in which animal experiments have been conducted without using the appropriate anaesthesia will not be accepted.

Manuscripts (including all figures and tables) must be submitted in English and in duplicate.

Text should not exceed 2-3 typewritten pages (50-60 lines). 1-2 relevant figures or tables. Summary of maximum 4 lines. Abbreviations should be properly explained. References should be numbered consecutively and be presented on a separate page. Name and address have to be placed directly under the title. Linguistically inadequate manuscripts will be returned. Footnotes should be avoided.

Figures Illustrations should be separate from the text. with the author's name on the back in soft pencil. The desired labelling should be shown on a second set of figures, which will be used as a model for inscriptions. Drawings for reproductions should be on good paper in Indian ink, photographs should be supplied as glossy positive prints. The illustrations should be at least one and a half times as large as the definitive size desired. Over-large figures can be easily damaged in the mail. Captions should be selfexplanatory, without reference to the text.

Tables should be provided with a title and with selfexplanatory captions.

Headings In submitting their manuscript to Experientia, authors are requested to indicate one of the headings mentioned below, under which they would wish to place their short communication:

1. Mathematics and Physics; 2. Cosmology, Astronautics, Cosmonautics; 3. Mineralogy, Geophysics, Oceanography; 4. Inorganic and Physical Chemistry; 5. Organic Chemistry; 6. Biophysics; 7. Molecular Biology, Cellular Biology; 8. Genetics; 9. Botany; 10. Zoology; 11. Ecology; 12. Biochemistry (analytic and synthetic); 13. Biochemistry (Enzymes, Metabolism); 14. Physiology; 15. Neurology; 16. Pharmacology, Toxicology, Pathology; 17. Experimental Gerontology; 18. Anatomy, Histology, Cytology, Histochemistry; 19. Embryology; 20. Endocrinology; 21. Circulation, Cardiology, Angiology; 22. Nutrition, Gastroenterology; 23. Hematology, Serology; 24. Immunology, Allergy; 25. Microbiology, Parasitology, Chemical Therapeutics; 26. Oncology, Carcinology, Cytostatics; 27. Radiology.

Reprints The authors receive 50 reprints, without cover, free of charge. Price-list for further reprints is available. 